\title{
Prognostic histological factors in patients with esophageal squamous cell carcinoma after preoperative chemoradiation followed by surgery
}

Cheng-Che Tu ${ }^{1,2,3}$, Po-Kuei Hsu ${ }^{1,2,5^{*}}$, Ling-I Chien ${ }^{4}$, Wan-Chen Liư ${ }^{4}$, Chien-Sheng Huang ${ }^{1,2}$, Chih-Cheng Hsieh ${ }^{1,2}$, Han-Shui Hsu $u^{1,2}$ and Yu-Chung $\mathrm{Wu}^{1,2}$

\begin{abstract}
Background: Pathological response is an important marker for tumor aggressiveness in patients with esophageal squamous cell carcinoma (ESCC) who receive preoperative chemoradiation followed by esophagectomy. We aim to evaluate the prognostic value of histological factors after trimodality treatments.

Methods: 91 patients who received preoperative chemoradiation followed by transthoracic esophagectomy between 2009 and 2014 were included. The pathological examination was reviewed. Overall survival and disease free survival were recorded. Survival analysis was performed using the Cox regression model, and the survival curves were compared by the log-rank test.

Results: Survival analysis showed lymphovascular invasion (LVI, hazard ratio [HR]: 2.009, $p=0.029$ ), perineural invasion (PNI, HR: 2.226, $p=0.019$ ), ypN stage (HR: 2.041, $p=0.019$ ), extracapsular invasion (ECI, HR: 2.804, $p=0.003$ ), and incomplete resection (HR: 1.897, $p=0.039$ ) as unfavorable prognostic factors affecting overall survival (OS). Moreover, tumor regression grade (TRG, HR: 1.834, $p=0.038$ ), LVI (HR: 1.975, $p=0.038$ ), ECI (HR: 2.836, $p=0.003$ ), and incomplete resection (HR: 2.254, $p=0.007$ ) adversely affected disease-free survival (DFS). Prognostic classification based on poor primary tumor (TRG2/3, $\mathrm{LVI}(+)$, and PNI (+)), lymph node $(y \mathrm{pN}(+)$ and $\mathrm{ECI}(+))$, and surgical (incomplete resection) factors significantly predicts OS $(p=0.013)$ and DFS $(p=0.017)$. However, the use of postoperative adjuvant therapy was not a significant prognostic factor even in medium- and high-risk ESCC patients who underwent trimodality treatments.
\end{abstract}

Conclusions: Histological factors, including primary tumor, lymph node, and surgical factors has high prognostic value for predicting outcomes in ESCC patients receiving preoperative chemoradiation followed by surgery.

Keywords: Adjuvant therapy, Esophageal cancer, Neoadjuvant therapy, Survival

\section{Background}

Trimodality treatments, which includes preoperative chemoradiation and surgery, is the approach recommended by the National Comprehensive Cancer Network (NCCN) clinical practice guidelines for most locally advanced esophageal cancers [1]. In the prospective randomized

\footnotetext{
* Correspondence: hsupokuei@yahoo.com.tw

${ }^{1}$ Division of Thoracic Surgery, Department of Surgery, Taipei Veterans General Hospital, Taipei, Taiwan

${ }^{2}$ School of Medicine, National Yang-Ming University, Taipei, Taiwan Full list of author information is available at the end of the article
}

CROSS (Chemoradiotherapy for Oesophageal Cancer Followed by Surgery Study) study, the median overall survival (OS) was 49.4 months in the preoperative chemoradiation plus surgery group vs. 24.0 months in the surgery alone group [2]. However, considerable prognostic differences resulting from varied response to chemoradiation have been observed in patients receiving trimodality treatment. Patients with a partial response to preoperative chemoradiation or no response at all were more likely to have disease recurrence than those with a complete response [3]. 
Moreover, pathologic non-responders to chemoradiation had no survival benefit when compared with patients who underwent only surgery [4-6]. To evaluate the prognostic impact of pathologic response and provide prognostic discrimination in esophageal cancer patients who received preoperative chemoradiation followed by surgery, a combined classification of primary tumor regression and lymph node status has been recently proposed by Holscher et al. [7] Although patients with a major response (defined by the presence of less than $10 \%$ of vital cells in the primary tumor) and ypNo had a 5-year survival rate of $64 \%$, the rate was only $18 \%$ in those with a minor response and $\mathrm{ypN}(+)$.

However, Holscher's prognostic classification was based on histological response in esophageal adenocarcinoma. Information regarding the prognostic impact of histological response in esophageal squamous cell carcinoma (ESCC) is limited in the literature. In this study, we aimed to evaluate the prognostic impact of histological factors in patients with ESCC after preoperative chemoradiation and esophagectomy.

\section{Methods}

We retrospectively reviewed the records of 91 consecutive ESCC patients who had undergone preoperative chemoradiation followed by transthoracic esophagectomy at the Taipei Veterans General Hospital between January 2009 and December 2014. Since patients with cervical ESCC would be treated with definitive chemoradiation, only patients with intrathoracic ESCC were included. Preoperative staging workup were previously described [8]. In particular, endoscopic ultrasound (EUS) was an optional procedure, but was required for confirmation of cT1 or cT2 lesions.

Preoperative chemoradiation included two courses of chemotherapy that were administered with a 4-week interval. The chemotherapy regimen included $80 \mathrm{mg} / \mathrm{m}^{2}$ of cisplatin administered intravenously on day 1 followed by continuous intravenous infusion of $600 \mathrm{mg} / \mathrm{m}^{2} 5$ fluorouracil (5-FU) and $90 \mathrm{mg} / \mathrm{m}^{2}$ leucovorin on days 1 through 4 concurrently with 45-50.4 Gy of externalbeam radiation with the dose per fraction of 1.8 to $2 \mathrm{~Gy}$ for primary tumors and mediastinal lymph node regions. The clinical target volume was defined as the gross target tumor volume delineated on CT scans and other diagnostic images along with $3-5-\mathrm{cm}$ cephalic and at least $5-\mathrm{cm}$ caudal margins. A chest CT scan was routinely performed after chemoradiation to determine the resectability.

Surgical resections were performed using the McKeown tri-incisional esophagectomy method. The surgical approaches used were minimally invasive esophagectomy (MIE, right-sided video-assisted thoracoscopic surgery [VATS] plus laparoscopic surgery) and hybrid esophagectomy (right-sided VATS plus laparotomy). The details of surgical procedures were previously described [8]. Pathological examination that was conducted according to the $7^{\text {th }}$ edition AJCC TNM staging system [9]. Tumor response was graded using the CAP (College of American Pathologist) Cancer Protocol for Esophageal Carcinoma [10]. Tumor regression grade (TRG) 0 (complete response) indicated no residual cancer cells. TRG 1 (moderate response) was defined as minimal residual cancer; TRG 2 (minimal response), as partial regression of the tumor; and TRG 3 (poor response) indicated that there was no definite response identified. The presence of lymphovascular invasion (LVI), perineural invasion (PNI), and extracapsular invasion (ECI) was recorded. The presence of tumor cells at proximal or distal cut end, a circumferential margin of less than $1 \mathrm{~mm}$, or M1 stage indicated incomplete resection.

Patients who were living after the operation were followed-up at our outpatient department. OS was defined as the time from the date of diagnosis until death or last known follow-up. Disease-free survival (DFS) was defined as the interval between surgical resection and the first detection of recurrence, death, or the last evaluation for recurrence. Patients who died due to surgery-related complications and those with M1 disease detected at the time of the surgery were excluded from the DFS analysis.

The Institutional Review Board of the Taipei-Veterans General Hospital approved this study and granted a waiver for the informed consent process.

\section{Statistical analysis}

The Pearson Chi-square test was used to compare categorical variables. The Student $t$-test and ANOVA were used for comparison of continuous variables. Survival curves, plotted by the Kaplan-Meier method, were compared by the log-rank test. The Cox regression model was utilized in prognostic factor analysis. All calculations were performed using Statistical Product and Service Solutions (version 17, SPSS Inc., Chicago, IL) and a twosided $p$-values $<0.05$ was considered significant.

\section{Results \\ Patient characteristics}

Table 1 shows the detailed clinical and pathological characteristics of the patients with ESCC as well as their prognostic relevance. The mean age of the patients was $55.4+/-9.1$ years. Four patients with clinical stage T1 tumors, but associate with clinical positive nodal involvement were given chemoradiation before esophagectomy. Five patients with ypM1 stage were because of incidentally identified metastasis (lung or liver) during esophagectomy. A total of 4 patients (4.4\%) died of 
Table 1 Patient demographics and the relevance to overall survival

\begin{tabular}{|c|c|c|c|c|}
\hline Variables & No. of patients & 3-years survival rate (\%) & Median survival, month (95\% Cl) & $p$ value \\
\hline Age (yrs) & & & & 0.144 \\
\hline$>55$ & 47 & 53.6 & $42.0(19.1-64.9)$ & \\
\hline$<=55$ & 44 & 39.5 & $26.0(17.2-34.8)$ & \\
\hline \multicolumn{5}{|l|}{ Sex } \\
\hline Male & 88 & 46.3 & $32.0(21.9-42.1)$ & 0.799 \\
\hline Female & 3 & 66.7 & $a^{a}$ & \\
\hline Location & & & & 0.136 \\
\hline Upper third & 24 & 35.4 & $15.0(0.0-36.6)$ & \\
\hline Middle third & 45 & 44.2 & $27.0(13.7-40.3)$ & \\
\hline Lower third & 22 & 64.5 & $-{ }^{a}$ & \\
\hline Differentiation & & & & 0.096 \\
\hline Grade I & 3 & 33.3 & $12.0(10.4-13.6)$ & \\
\hline Grade II & 42 & 44.1 & $27.0(12.6-41.4)$ & \\
\hline Grade III & 11 & 37.9 & $11.0(8.1-13.9)$ & \\
\hline N/A & 35 & 57.0 & $-{ }^{a}$ & \\
\hline Tumor size $(\mathrm{cm})$ & & & & 0.665 \\
\hline$<2.5$ & 51 & 39.8 & $27.0(13.4-40.6)$ & \\
\hline$>=2.5$ & 40 & 54.4 & $37.0(17.3-56.7)$ & \\
\hline CT stage & & & & 0.918 \\
\hline $\mathrm{T} 1$ & 4 & 50.0 & - & \\
\hline $\mathrm{T} 2$ & 17 & 51.5 & $37.0(21.0-53.0)$ & \\
\hline T3 & 66 & 45.7 & $27.0(12.1-41.9)$ & \\
\hline $\mathrm{T} 4$ & 4 & 50.0 & $-^{a}$ & \\
\hline cN stage & & & & 0.778 \\
\hline No & 27 & 36.5 & $32.0(22.3-41.7)$ & \\
\hline N1 & 24 & 54.8 & $37.0(9.06-64.9)$ & \\
\hline N2 & 17 & 41.0 & $21.0(8.6-33.4)$ & \\
\hline N3 & 13 & 27.2 & $24.0(17.4-30.6)$ & \\
\hline ypT stage & & & & 0.908 \\
\hline TO & 32 & 53.4 & $37.0(14.6-59.4)$ & \\
\hline Tis/1 & 11 & 42.4 & $25.0(6.3-43.7)$ & \\
\hline $\mathrm{T} 2$ & 18 & 54.0 & $37.0(15.5-58.5)$ & \\
\hline T3 & 24 & 34.1 & $27.0(14.0-40.0)$ & \\
\hline T4 & 6 & 44.4 & $14.0(2.2-25.8)$ & \\
\hline ypN stage & & & & $0.032+$ \\
\hline No & 56 & 53.5 & $37.0(21.3-52.7)$ & \\
\hline $\mathrm{N} 1$ & 20 & 50.4 & $37.0(2.0-72.0)$ & \\
\hline N2 & 12 & 13.0 & $15.0(12.0-18.0)$ & \\
\hline N3 & 3 & 33.0 & $24.0(0.0-49.6)$ & \\
\hline ypM stage & & & & $0.019+$ \\
\hline MO & 86 & 49.1 & $35.0(23.8-46.2)$ & \\
\hline M1 & 5 & 0.0 & $13.0(0.1-25.9)$ & \\
\hline ypStage & & & & $0.031+$ \\
\hline 0 & 25 & 63.8 & $-a^{a}$ & \\
\hline
\end{tabular}


Table 1 Patient demographics and the relevance to overall survival (Continued)

\begin{tabular}{|c|c|c|c|c|}
\hline I & 7 & 42.9 & $25.0(0.0-58.4)$ & \\
\hline$\|$ & 30 & 52.2 & $37.0(20.5-53.5)$ & \\
\hline III & 24 & 34.9 & $16.0(7.5-24.5)$ & \\
\hline IV & 5 & 0.0 & $13.0(0.1-25.9)$ & \\
\hline TRG & & & & 0.454 \\
\hline 0 & 29 & 57.9 & 37.0 & \\
\hline 1 & 32 & 48.2 & $25.0(5.8-44.3)$ & \\
\hline 2 & 15 & 17.6 & $27.0(12.4-41.6)$ & \\
\hline 3 & 15 & 45.0 & $26.0(3.6-48.4)$ & \\
\hline LVI & & & & $0.024+$ \\
\hline No & 70 & 55.0 & $37.0(26.3-47.7)$ & \\
\hline Yes & 21 & 23.4 & $14.0(8.9-19.1)$ & \\
\hline $\mathrm{PNI}$ & & & & $0.014 \dagger$ \\
\hline No & 76 & 55.2 & $37.0(26.1-47.9)$ & \\
\hline Yes & 15 & 15.6 & $13.0(9.4-16.6)$ & \\
\hline $\mathrm{ECl}$ & & & & $0.002+$ \\
\hline No & 77 & 50.5 & $37.0(23.9-50.1)$ & \\
\hline Yes & 14 & 23.9 & $11.0(7.9-14.1)$ & \\
\hline Total resected lymph node number & & & & 0.134 \\
\hline$<18$ & 44 & 55.8 & $37.0(21.4-52.6)$ & \\
\hline$>=18$ & 47 & 38.2 & $23.0(10.6-35.4)$ & \\
\hline Incomplete resection & & & & $0.033+$ \\
\hline No & 63 & 55.0 & $37.0(26.1-47.9)$ & \\
\hline Yes & 28 & 26.0 & $21.0(9.5-32.5)$ & \\
\hline Surgical approach & & & & 0.950 \\
\hline MIE & 62 & 47.9 & $35.0(22.0-48.0)$ & \\
\hline Hybrid & 29 & 30.7 & $27.0(15.0-39.0)$ & \\
\hline Adjuvant therapy & & & & 0.434 \\
\hline No & 67 & 47.4 & $32.0(18.9-45.1)$ & \\
\hline Yes & 24 & 42.4 & $35.0(19.2-50.8)$ & \\
\hline
\end{tabular}

$\mathrm{Cl}$ confidence interval, $T R G$ tumor regression grade, $L V I$ lymphovascular invasion, $P N /$ perineural invasion, $E C l$ extracapsular invasion, MIE minimally invasive esophagectomy and reconstruction; ${ }^{\mathrm{a}}$ :median survival not reached; + : $p<0.05$

surgery-related complications. The mean follow-up time for the entire cohort was 22.0 months (range: 3 to 67 months). The median OS was 32 months (95\% confidence interval [CI], 21.9-42.1 months), with 1-year and 3 -year OS rates of 78.7 and $46.5 \%$, respectively. The median DFS was 11 months (95\% CI, 4.5-17.5 months), with 1-year and 3-year DFS rates of 49.1 and 27.5\%, respectively. Survival according to the AJCC staging is shown in Fig. 1a.

\section{Prognostic value of histological factors}

In the Cox regression model, we did not include $M$ stage into the analysis because it was one of the characteristics of incomplete resection. The univariate survival analysis is shown in Table 2. Factors adversely affecting OS included LVI, PNI, ypN stage, ECI, and incomplete resection. Unfavorable prognostic factors for DFS included LVI, ECI, incomplete resection, and TRG. Since some of these factors were highly correlated (for example, TRG was significantly correlated with LVI and PNI; and ypN stage was correlated ECI), we did not perform multivariate analysis, trying to avoid the problem of multicollinearity in a multiple regression model. Of these factors, TRG, LVI, and PNI were the primary tumor features, ypN stage and ECI were the lymph node features, and resection margins were the surgical features. Patients 

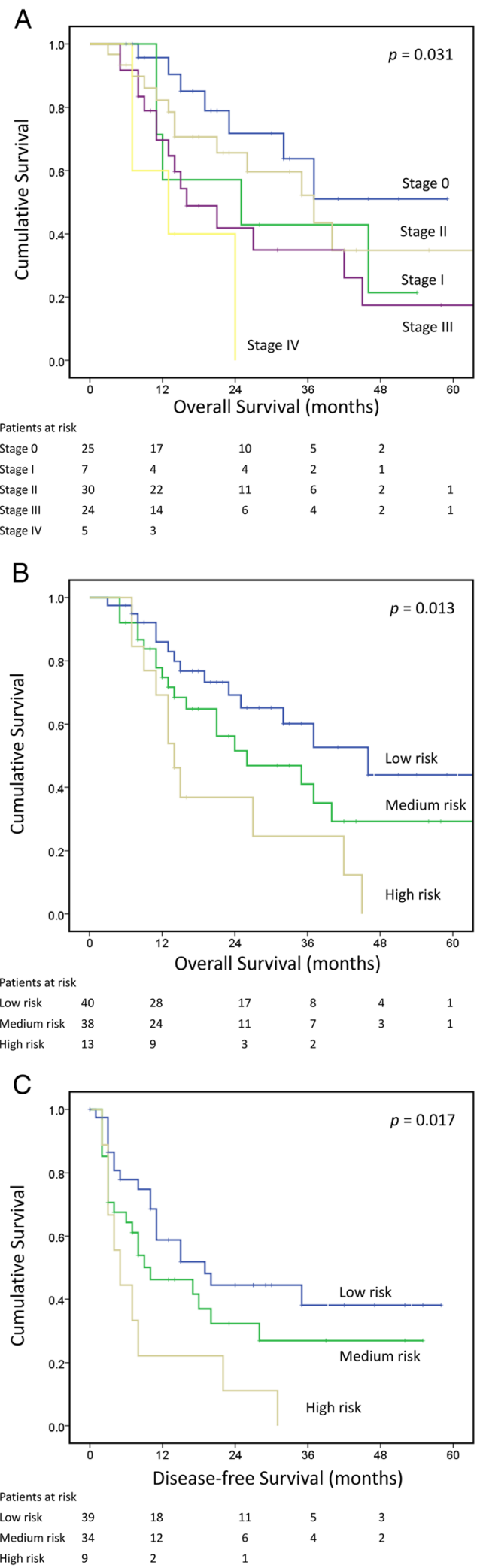

Furvival curves according to AJCC staging system (a) and ou own classification (b overall survival; c disease free survival). The curves were plotted with Kaplan-Meier method and compared with log-rank test

with any of TRG2/3, LVI(+) or PNI(+) were characterized as having poor primary tumor factors, whereas patients with either $\mathrm{ypN}(+)$ or ECI $(+)$ were regarded as having poor lymph node factors. Patients with incomplete resection were considered as ha ving poor surgical factors. The classification was based on the number of poor histological factors and had a range from 0 to 3 (Table 3). Using Cox regression model, we demonstrated that these factors have additional effect; which means, patients with 1,2 , and 3 poor factors had HR of 1.576, 1.728 , and 3.130 compared to those without any poor factor in the overall survival analysis $(p=0.048)$. Patients with 1, 2, and 3 poor factors had HR of 1.062, 2.030, and 2.958 compared to those without any poor factor in the disease-free survival analysis $(p=0.027)$. We then classified patients with 0 poor histological factors as low risk, those with 1 or 2 factors as medium risk, and patients with 3 factors as high risk. In all, there were 40, 38, and 13 patients in the low-, medium-, and high-risk groups, respectively. The 3 -year OS was $60.1,41.0$, and $24.6 \%$ in the low-, medium-, and high-risk groups, respectively. The median survival (95\% CI) was 46.0 (25.7-66.3), 26.0 (8.4-43.6), and 14.0 (10.6-17.4) months in the low-, medium-, and high-risk groups, respectively $(p=0.013$, Fig. 1b). The 3-year DFS was 38.1, 27.0, and $0.0 \%$ in low-, medium-, and high-risk groups, respectively. The median DFS survival $(95 \% \mathrm{CI})$ was 19.0 (8.0-30.0), 10.0 (0.8-19.2), and 5.0 (2.1-7.9) months in the low-, medium-, and high-risk groups, respectively $(p=0.017$, Fig. 1c).

\section{Impact of adjuvant therapy on patients with predicted poor outcomes}

We further evaluated the survival impact of adjuvant therapy in ESCC patients predicted to have worse outcomes after trimodality therapy. Of all the patients in the medium- or high-risk groups, 23 received adjuvant therapy whereas 28 received observation only. Although there seemed to be a trend towards better OS in patients receiving adjuvant therapy, no statistical significance was reached; the 1- and 3-year OS rates were 95.5 and 39.5\% in patients receiving adjuvant treatment and 54.8 and $32.7 \%$ in those receiving observation only (Fig. $2 \mathrm{a}, p=$ 0.052). No significant difference was found between the two groups in terms of DFS. The 1- and 3-year DFS rates were 37.4 and $23.4 \%$ in patients receiving adjuvant treatment and 51.4 and $17.1 \%$ in those receiving observation only (Fig. $2 \mathrm{~b}, p=0.824$ ). On the basis of our observations, it is suggested that adjuvant therapy did not 
Table 2 Prognostic factors for overall survival (OS) and disease free survival (DFS)

\begin{tabular}{|c|c|c|c|c|}
\hline \multirow{3}{*}{ 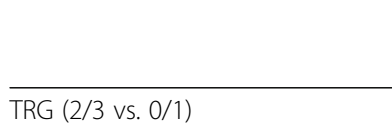 } & \multicolumn{2}{|l|}{ OS } & \multicolumn{2}{|l|}{ DFS } \\
\hline & HR $(95 \% \mathrm{Cl})$ & $p$ value & $\mathrm{HR}(95 \% \mathrm{Cl})$ & $p$ value \\
\hline & $1.455(0.801-2.645)$ & 0.218 & $1.834(1.035-3.248)$ & $0.038^{*}$ \\
\hline LVI (yes vs. no) & $2.009(1.076-3.752)$ & $0.029^{*}$ & $1.975(1.040-3.752)$ & $0.038^{*}$ \\
\hline PNI (yes vs. no) & $2.226(1.144-4.331)$ & $0.019^{*}$ & $1.481(0.693-3.164)$ & 0.311 \\
\hline ypN stage (+ vs. - ) & $2.041(1.123-3.708)$ & $0.019^{*}$ & $1.628(0.926-2.861)$ & 0.090 \\
\hline $\mathrm{ECl}$ (yes vs. no) & $2.804(1.404-5.599)$ & $0.003^{*}$ & $2.836(1.401-5.740)$ & $0.003^{*}$ \\
\hline Incomplete resection (yes vs. no) & $1.897(1.033-3.482)$ & $0.039^{*}$ & $2.254(1.248-4.071)$ & $0.007^{*}$ \\
\hline
\end{tabular}

$N=91$ and 82 for OS and DFS analysis, respectively

$H R$ hazard ratio, $C l$ confidence interval, TRG tumor regression grade, $L V I$ lymphovascular invasion, $P N I$ perineural invasion, ECl extracapsular invasion; *: $p<0.05$ by Univariate Cox regression analysis

have a significant effect on survival in patients with predicted poor outcome.

\section{Discussion}

The current $7^{\text {th }}$ AJCC staging system was based on the retrospective analysis of patients who underwent surgery without induction treatment or adjuvant therapy [9]. The prognostic value of such pathologic staging for patients who received preoperative treatment is questionable [11-13]. For example, Kim et al. have shown that the staging system was not very predictive of survival in patients after preoperative treatment. In particular, there was less distinctiveness among stage subgroups. Supplementation of the AJCC TNM staging system with pathologic response for a better prediction of patients' outcomes has been proposed for a long time [14]. Indeed, the pathological response of the tumor is a critical determinant of survival in patients receiving neoadjuvant treatment. Although chemoradiation responders have superior survival, the 5 -year survival is only $18-27 \%$ in poor responders $[3-6,15,16]$. Some authors reported that non-responders to chemoradiation received no benefit and had even worse survival compared to patients treated with primary esophagectomy [4-6]. By combining classifications of primary tumor regression and lymph node status, Hölscher et al. have established a 3-grade classification with a good performance in prognostic discrimination [7]. However, Holscher's prognostic classification was based on histological response in esophageal adenocarcinoma. Information regarding the prognostic classification of histological response in ESCC is limited in the literature. In the present study, we demonstrated the prognostic value of histological parameters including TRG, LVI, PNI, ypN stage, ECI, as well as incomplete resection, in ESCC. In addition to the well-known poor prognostic factors, such as TRG, ypN, and incomplete resection, our prognostic classification also includes LVI, PNI, as well as ECI. Schoppmann et al. have reported that both the 5-year OS $(14 \%$ vs. $60 \%, p<0.001)$ and the 5 -year DFS $(14 \%$ vs. $49 \%, p<0.001)$ were significantly reduced in patients with positive LVI [17]. Chen et al. have also reported that ESCC patients with PNI-negative tumors had a 1.7fold increase in the 5-year recurrence-free survival over the 5-year DFS for with patients with PNI-positive tumors [18]. As for ECI, D'Annoville reported that the proportion of ECI detected in N1, N2, and N3 patients was $28 \%$ (21 of 73 patients), $51 \%$ (21 of 41 patients), and $70 \%$ (17 of 24 patients), respectively. The presence of ECI seems to have negative additive impact on DFS, regardless of the $\mathrm{pN}$ stage [19]. ECI detected after preoperative chemoradiation and esophagectomy have been reported to be associated with a very dismal prognosis. In D'Journo's study, the 5-year DFS rates were $46 \%$ in N0 patients, $36 \%$ in $\mathrm{N}+$ with intracapsular invasion patients, and $11 \%$, in $\mathrm{N}+$ with ECI patients [20].

As the information provided by these histological factors can identify high-risk patients, the requirement of more aggressive treatment for the patients with predicted poor outcomes has to be addressed; however, the effect of adjuvant therapy in these patients is doubtful. Only a few studies have evaluated the effect of adjuvant therapy in patients who underwent trimodality treatment; this is partially because postoperative chemotherapy or chemoradiation is poorly tolerated in esophageal cancer patients. For example, $46 \%$ of patients in Stile's

Table 3 Category of each ominous factors (primary tumor, lymph node and surgical) in our classification and the parameters in each category

\begin{tabular}{llll}
\hline Category & Tumor factor & Lymph node factor & Surgical factor \\
\hline$(+)$ if any of these parameters & TRG $(2 / 3)$ & ypN stage $(+)$ & Incomplete resection \\
& LVI $(+)$ & ECI $(+)$ & \\
& PNI $(+)$ & & \\
\hline
\end{tabular}




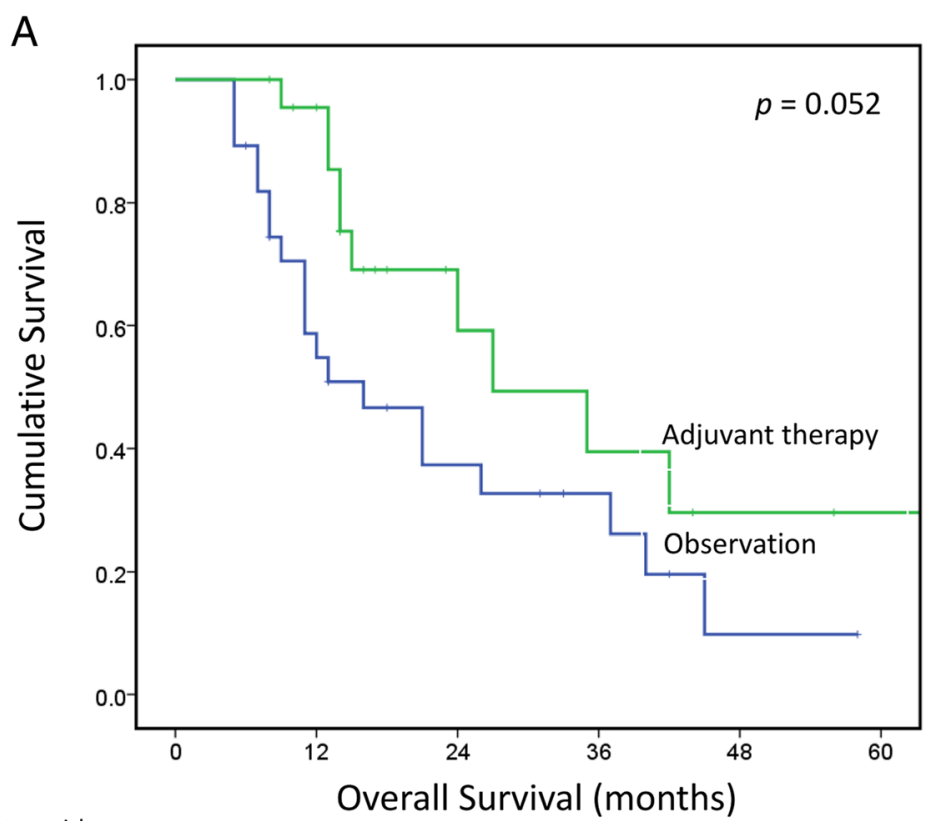

$\begin{array}{llllll}\text { Patients at risk } & & & & & \\ \text { Adjuvant therapy } & 23 & 19 & 6 & 4 & 2 \\ \text { Observation } & 28 & 14 & 8 & 5 & 1\end{array}$

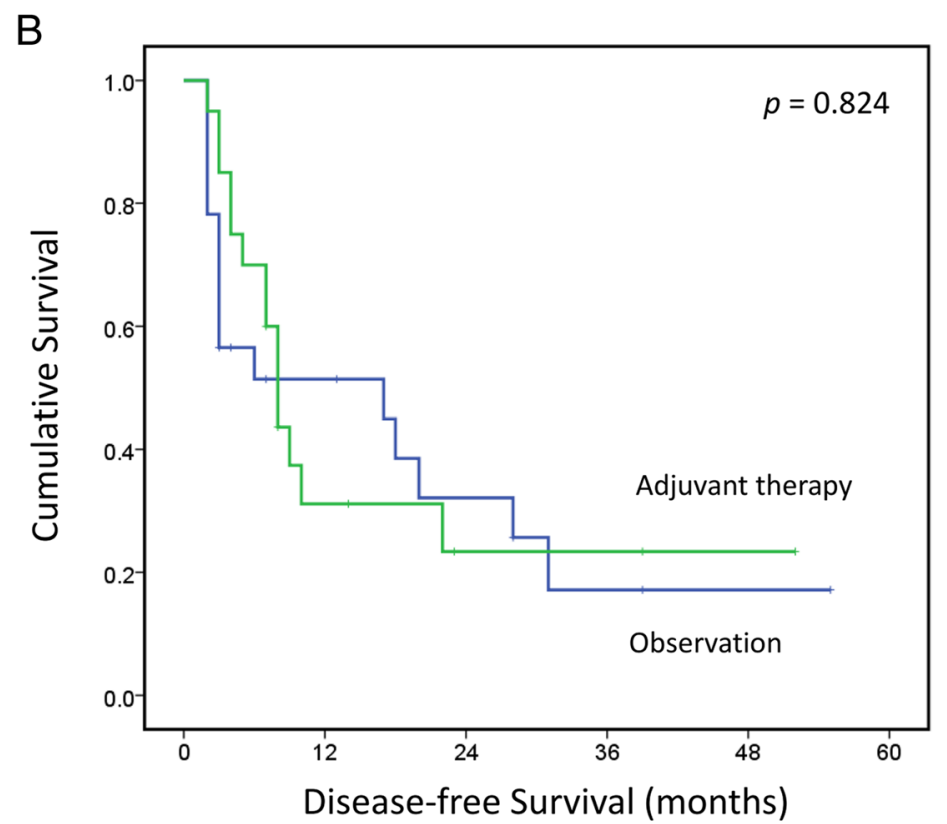

Patients at risk

$\begin{array}{llllll}\text { Adjuvant therapy } & 20 & 5 & 2 & 2 & 1 \\ \text { Observation } & 23 & 9 & 5 & 2 & 1\end{array}$

Fig. 2 Overall survival (a) and disease free survival (b) of medium/high risk patients with (green line) or without (blue line) adjuvant therapy. The curves were plotted with Kaplan-Meier method and compared with log-rank test

study received adjuvant chemotherapy, and most of the (89\%) received only one cycle [21]. In the current study, 24 patients $(26.4 \%)$ received adjuvant therapy: 11 of them received adjuvant chemotherapy and 13 of them received adjuvant chemoradiation. Of all patients, 18
(75\%) completed the treatment. Out of 6 cases of incomplete treatment, 5 were due to disease progression, and therefore, palliative chemotherapy was administered to the patients instead. The sixth patient had pneumonia with septic shock during the second course of 
chemotherapy. High completion rates of adjuvant therapy in this study may be related to the use of minimally invasive surgical techniques that resulted in earlier recovery from surgical trauma and faster return to baseline physical function, which, in turn, improved the delivery of adjuvant therapy. However, in our series, there was no significant difference in OS or DFS between patients with or without adjuvant therapy, which was compatible with the results from the literature. In the study by Meredith et al., which included 301 patients with esophageal adenocarcinoma (86.7\%) and 46 patients with ESCC (13.3\%), the 5-year OS and DFS were 43 and 43\%, respectively, in patients who were treated with adjuvant therapy and 46 and $48 \%$, respectively, in those who were not [6]. In their study, 34 of 262 patients (13.0\%) received adjuvant therapy after preoperative treatment and esophagectomy. They found that adjuvant therapy had no impact on survival outcomes in patients who received trimodality treatment. As echoed in Meredith's study, adjuvant therapy was not a predictor of survival in another study by Stiles et al. [21].

Our study has some limitations. First, EUS was not performed in every patient. Some patients came to us with intolerable dysphagia and near total endoscopic obstruction, which made EUS infeasible. In such cases, CT scan or bronchoscopy determined the cT3 and CT4 lesions. Second, data on tumor differentiation, which was determined by pretreatment diagnostic biopsy, was lacking for a large portion of patients. Some patients were referred to us after being diagnosed elsewhere, and we did not have an access to the biopsy specimens. Third, the small number of patients in this study limits the ability of the new classification to predict survival with significant difference between groups. Furthermore, most patients in this cohort were men, which, in agreement with the results from our nation-wide database, confirms that ESCC is the male-predominant disease in Taiwan [22]. Whether our prognostic classification can be applied to female esophageal cancer patients remains to be elucidated. Finally, although the present study is one of the few studies examining the effect of adjuvant therapy in patients who received trimodality treatment for esophageal cancer and is probably the first one discussing this topic exclusively in terms of squamous cell carcinoma histology, the sample size was small, and the assignment of patients to adjuvant therapy or observation groups was not randomized and subject to substantial bias. In general, the main indications for adjuvant therapy include positive lymph nodes, close margins, incomplete resection, and the absence of postoperative complications. We believe that further large-scale study is needed to validate our findings and determine the role of adjuvant therapy in high-risk patients.

\section{Conclusions}

Histological factors including primary tumor factor (TRG, LVI, and PNI), lymph node factor (ypN stage and ECI), and surgical factor (incomplete resection) has a significant value in predicting survival in patients after trimodality treatments. In the future, it will be essential to establish a surveillance protocol to determine the role of adjuvant therapy in high-risk patients.

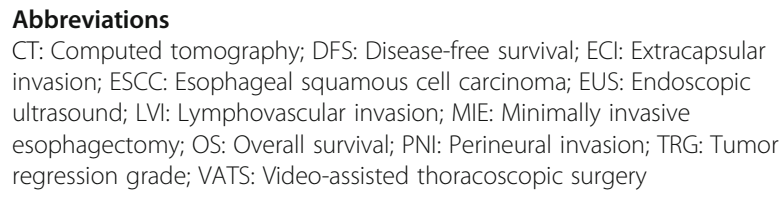
invasion; ESCC: Esophageal squamous cell carcinoma; EUS: Endoscopic ultrasound; LVI: Lymphovascular invasion; MIE: Minimally invasive esophagectomy; OS: Overall survival; PNI: Perineural invasion; TRG: Tumor regression grade; VATS: Video-assisted thoracoscopic surgery

\section{Acknowledgements}

We thank the Taipei Veterans General Hospital Esophageal Cancer Panel Members, including Mei-Han Wu, Chun-Ku Chen, Hsiao-Ping Chou in Department of Radiology; Shyh-Jen Wang, Ko-Han Lin in Department of Nuclear Medicine; Teh-Ying Chou, Lei-Chi Wang in Department of Pathology; ChuehChuan Yen, Ming-Huang Chen, Sheng-Yu Chen, Pin-I Huang, Yi-Wei Chen in Department of Oncology, for reviewing patient's examination results and arranging patient's treatments and follow-up.

Funding

None.

\section{Availability of data and materials}

The datasets during and/or analyzed during the current study available from the corresponding author on reasonable request and IRB approval.

\section{Authors' contributions}

TCC: drafting, conception, design of the work, analysis and interpretation of data. HPK: drafting, conception and design of the work, analysis and interpretation of data. CLI: data acquisition and analysis. LWC: data acquisition. HCS: conception of the work and interpretation of data. HCC conception of the work and interpretation of data. HHS: interpretation of data and final approval. WYC: interpretation of data and final approval. All authors have read and approved the manuscript.

\section{Competing interests}

The authors declare that they have no competing interests.

\section{Consent for publication}

N/A.

\section{Ethics approval and consent to participate}

The Institutional Review Board of the Taipei Veterans General Hospital approved this study and granted a waiver for the informed consent process.

\section{Author details}

'Division of Thoracic Surgery, Department of Surgery, Taipei Veterans General Hospital, Taipei, Taiwan. ${ }^{2}$ School of Medicine, National Yang-Ming University, Taipei, Taiwan. ${ }^{3}$ Division of Thoracic Surgery, Department of Surgery, Chang Bing Show Chwan Memorial Hospital, Changhua, Taiwan. ${ }^{4}$ Department of Nursing, Taipei Veterans General Hospital, Taipei, Taiwan. ${ }^{5}$ Division of Thoracic Surgery, Department of Surgery, Taipei-Veterans General Hospital, No. 201, Sec. 2, Shih-Pai Road, Taipei, Taiwan.

Received: 26 July 2016 Accepted: 12 January 2017

Published online: 19 January 2017

References

1. National Comprehensive Cancer Network. Esophageal cancer clinical practice guidelines in oncology. Available at:http://www.nccn.org/. Accessed 31 Dec 2016. 
2. van Hagen P, Hulshof MC, van Lanschot JJ, Steyerberg EW, van Berge Henegouwen $\mathrm{Ml}$, et al. Preoperative chemoradiotherapy for esophageal or junctional cancer. N Engl J Med. 2012;366:2074-84.

3. Meguid RA, Hooker CM, Taylor JT, Kleinberg LR, Cattaneo SM, Sussman MS, et al. Recurrence after neoadjuvant chemoradiation and surgery for esophageal cancer: does the pattern of recurrence differ for patients with complete response and those with partial or no response? J Thorac Cardiovasc Surg. 2009;138:1309-17.

4. Dittrick GW, Weber JM, Shridhar R, Shridhar R, Hoffe S, Melis M, et al. Pathologic non responders after neoadjuvant chemoradiation for esophageal cancer demonstrated no survival benefit compared with patient treated with primary esophagectomy. Ann Surg Oncol. 2012;19:1678-84.

5. Hölscher AH, Bollschweiler E, Bogoevski D, Schmidt H, Semrau R, Izbicki JR. Prognostic impact of neoadjuvant chemoradiation in CT3 oesophagea cancer - a propensity score matched analysis. Eur J Cancer. 2014;50:2950-7.

6. Meredith KL, Weber JM, Turaga KK, Siegel EM, McLoughlin J, Hoffe S, et al. Pathologic response after neoadjuvant therapy is the major determinant of survival in patients with esophageal cancer. Ann Surg Oncol. 2010;17:1159-67.

7. Hölscher AH, Drebber U, Schmidt H, Bollschweiler E. Prognostic classification of histopathologic response to neoadjuvant therapy in esophageal adenocarcinoma. Ann Surg. 2014;260:779-84.

8. Hsu PK, Huang CS, Wu YC, Chou TY, Hsu WH. Open versus thoracoscopic esophagectomy in patients with esophageal squamous cell carcinoma. World J Surg. 2014;38:402-9.

9. Edge SB, Byrd DR, Compton CC, Fritz AG, Greene FL, Trotti A. American Joint Committee on Cancer (AJCC) cancer staging manual. 7th ed. Chicago: Springer, Inc.; 2010.

10. Washington K, Berlin J, Branton P, Burgart LJ Carter DK, Fitzgibbons P, et al. Protocol for the examination of specimens from patients with carcinoma of the esophagus. College of American Pathologists Cancer Protocols 2009;1-16 (available at http://www.cap.org/). Accessed 31 Dec 2016.

11. Davies AR, Gossage JA, Zylstra J, Mattsson F, Lagergren J, Maisey N, et al. Tumor stage after neoadjuvant chemotherapy determines survival after surgery for adenocarcinoma of the esophagus and esophagogastric junction. J Clin Oncol. 2014;32:2983-90.

12. Nomura M, Shitara K, Kodaira T, Hatooka S, Mizota A, Kondoh C, et al. Prognostic impact of the 6th and 7th American Joint Committee on Cancer TNM staging systems on esophageal cancer patients treated with chemoradiotherapy. Int J Radiat Oncol Biol Phys. 2012;82:946-52.

13. Kim JY, Nelson RA, Kim J, Raz D. How well does pathologic stage predict survival for esophageal adenocarcinoma after neoadjuvant therapy? J Thorac Dis. 2015:7:734-9.

14. Swisher SG, Hofstetter W, Wu TT, Correa AM, Ajani JA, Komaki RR, et al. Proposed Revision of the Esophageal Cancer Staging System to Accommodate Pathologic Response (pP) Following Preoperative Chemoradiation (CRT). Ann Surg. 2005:241:810-20.

15. Donahue JM, Nichols FC, Li Z, Schomas DA, Allen MS, Cassivi SD, et al. Complete pathologic response after neoadjuvant chemoradiotherapy for esophageal cancer is associated with enhanced survival. Ann Thorac Surg. 2009:87:392-8.

16. Berger AC, Farma J, Scott WJ, Freedman G, Weiner L, Cheng JD, et al. Complete response to neoadjuvant chemoradiotherapy in esophageal carcinoma is associated with significantly improved survival. J Clin Oncol. 2005;23:4330.

17. Schoppmann SF, Jesch B, Zacherl J, Riegler MF, Friedrich J, Birner P. Lymphangiogenesis and lymphovascular invasion diminishes prognosis in esophageal cancer. Surgery. 2013;153:526-34.

18. Chen JW, Xie JD, Ling YH, Li P, Yan SM, Xi SY, et al. The prognostic effect of perineural invasion in esophageal squamous cell carcinoma. BMC Cancer. 2014;14:313.

19. D'Annoville T, D'Journo XB, Loundou A, Trousse D, Dahan L, Doddoli C, et al. Prognostic impact of the extracapsular lymph node involvement on diseasefree survival according to the 7th edition of American Joint Committee on Cancer Staging System. Eur J Cardiothorac Surg. 2013;44:e207-11.

20. D'Journo XB, Avaro JP, Michelet P, Trousse D, Tasei AM, Dahan L, et al. Extracapsular lymph node involvement is a negative prognostic factor after neoadjuvant chemoradiotherapy in locally advanced esophageal cancer. J Thorac Oncol. 2009;4:534-9.
21. Stiles BM, Christos P, Port JL, Lee PC, Paul S, Saunders J, et al. Predictors of survival in patients with persistent nodal metastases after preoperative chemotherapy for esophageal cancer. J Thorac Cardiovasc Surg. 2010;139:387-94.

22. Hsu PK, Chen HS, Wu SC, Wang BY, Liu CY, Shih CH, et al. Impact of hospital volume on long-term survival after resection for oesophageal cancer: a population-based study in Taiwan. Eur J Cardiothorac Surg. 2014:46:e127-35

\section{Submit your next manuscript to BioMed Central and we will help you at every step:}

- We accept pre-submission inquiries

- Our selector tool helps you to find the most relevant journal

- We provide round the clock customer support

- Convenient online submission

- Thorough peer review

- Inclusion in PubMed and all major indexing services

- Maximum visibility for your research

Submit your manuscript at www.biomedcentral.com/submit
) Biomed Central 